\title{
Selective Mobile Communication within a Coverage Area Bounded by Radiating Cables
}

\author{
Hatim Behairy, ${ }^{1}$ Waleed Alrobian, ${ }^{1}$ Adnan Alghammas, ${ }^{1}$ Amr Alasaad, ${ }^{1}$ Brian Suter, \\ Mohammed Alshareef, ${ }^{1}$ and Yahya Alsuwayyeh ${ }^{1}$ \\ ${ }^{1}$ National Center for Electronics and Photonics Technology, King Abdulaziz City for Science and Technology, \\ Riyadh 11442, Saudi Arabia \\ ${ }^{2}$ Tellumat (Pty) Ltd., Cape Town 7966, South Africa
}

Correspondence should be addressed to Hatim Behairy; hbehairy@kacst.edu.sa

Received 16 August 2015; Revised 10 November 2015; Accepted 17 November 2015

Academic Editor: Peter Brida

Copyright (C) 2015 Hatim Behairy et al. This is an open access article distributed under the Creative Commons Attribution License, which permits unrestricted use, distribution, and reproduction in any medium, provided the original work is properly cited.

\begin{abstract}
It is often desirable to control mobile phone services in areas where complete silence is either expected or mandatory, including schools, places of worship, hospitals, and prisons. In contrast to conventional techniques, such as jammers or Faraday cages, we present a novel technique to selectively control mobile phone services within a desired area. Our solution enables the area's keeper to allow mobile phones on a whitelist to freely use mobile services without disruption while denying services to all other mobile phones that are within the boundaries of the desired area to be controlled. Our solution uses a base station controller to identify all mobile devices located within the area to be controlled, while an antenna is placed inside the area to attract all mobile devices in the area to connect to the base station controller. In previous work, we proposed a system that uses directional antennas for the attraction technique. In this work, we show that replacement of the directional antennas with a leaky feeder antenna enables more accurate control of mobile phone services in and around the area to be controlled. Simulations and experiments of the leaky feeder technique confirm its precise control of mobile phone services within the desired areas.
\end{abstract}

\section{Introduction}

In the modern always-connected lifestyle, almost everybody carries a mobile communication device of some sort, such as a conventional or a smart phone. This massive adoption of cellular service devices means that new social, security, and etiquette-related complexities have appeared in areas where silence is expected or even mandatory, such as in schools, universities, places of worship, hospitals, and prisons. In some environments, the use of mobile phones is undesirable, such as meeting rooms, places of worship, and hospitals, while their use in security-sensitive areas must be controlled according to a security plan [1-3].

The problem of silencing mobile phones in specific areas has been studied only briefly in the literature, including the review provided in [3]. To the best of our knowledge, no practical solutions have been proposed and implemented to date that do not require changes at either the network level or the Mobile Station (MS) level.
In existing mobile communication networks, the control of mobile phone services can be achieved in a number of ways with various levels of effectiveness:

(1) Complete control at all times of any service on any segment of the network by the Mobile Network Operator (MNO).

(2) Cooperative control, where the mobile phone user willingly activates/deactivates features on the phone in accordance with the regulations or recommendations for their current environment.

(3) Shielding using a Faraday cage or a Faraday shield [4].

(4) The use of Radio Frequency (RF) jammers.

In [5-7], several methods have been proposed to address this problem that mostly focus on suppression of the sounds on the phone, but no or very little effort has been made to control the services provided to the cellular phone based on either 
the location or time-based zones, which is the subject of this paper.

In our previous work [8-10], we proposed a system to selectively control mobile phone services in specific areas. The chosen mobile service can be controlled based on the identity of the user on a whitelist basis, where the whitelist is defined as a list of MSs that are allowed certain services in the controlled area (CA), while all other MSs that are in the $\mathrm{CA}$ and not in the whitelist will be denied certain services at specific times during the day (e.g., during scheduled prayer times at a place of worship) or at a specific location, such as a meeting room. The system consists of two virtual Base Transceiver Stations (vBTSs), each of which is connected to a directional antenna. The vBTS is used in this system configuration to mimic the operation of a conventional Base Transceiver Station (BTS). The first antenna is installed at the gate of the CA and points outward from the CA and is thus called the outer antenna, while the other antenna is also installed at the gate to the CA but points inward towards the CA and is thus called the inner antenna. The motivation behind this design is to detect the identity of an MS when it passes through the gate to the area by triggering the following sequence of events. If the MS is detected first by the outer antenna and then by the inner antenna, it is then considered to be within the CA and is subject to the set of mobile service rules imposed by the keeper of the area. When the controlled MS leaves the CA, it will be detected by the outer antenna again and will then be released from the system.

To force each MS that passes through the gate entering to the CA to connect to the two vBTSs installed in the CA, a signal that is stronger than the signal the MS is receiving from its current BTS is transmitted by the two antennas. This will attract the MS to connect to the vBTS, which in turn forces the MS to transmit its International Mobile Station Equipment Identity (IMEI) and International Mobile Subscriber Identity (IMSI) information to identify itself and camp on the vBTS. This enables the vBTS to have complete control over the MS. In particular, the vBTS will match the IMEI and IMSI to its data base. If the MS is not in the whitelist, it will hold on to that MS and no service is provided; while if the MS is in the whitelist, the vBTS sends a deny service message to that MS. When the MS receives this message, it will connect to another BTS and get the service from its MNO [8].

Two shortcomings were observed when using the directional antennas in this system architecture. The first is the unpredictable spillage of RF power to areas beyond the CA boundaries. The second is that RF signal transmission from the inner antenna causes signal reflections inside the CA. The first shortcoming leads to undesired control of MSs that happen to be near the CA. The second shortcoming leads to multipath fading, which in turn leads to attenuation of the radio signal power received by MSs in certain locations within the CA. When this phenomenon happens at a certain instant in time and at specific locations, an MS may receive a signal power from the vBTS weaker than the signal received from the MNO BTS. This will cause MS to undesirably camp on the MNO BTS, thus releasing it from the control of our system.

In this paper, we address the above shortcomings by proposing a simplification of the architecture that was presented in [8] and by replacing the two directional antennas with a Distributed Antenna System (DAS) that consists of a number of radiating antennas (which are often called leaky feeders) inside the CA [11]. A leaky feeder is characterized by its near field, and its transmitted signal power falls off quickly with distance, allowing for more localized control over the CA [12].

Operating transmitters designed to jam or block wireless communications (Active Jammers) is a violation of the law in many countries [13-17]. Passive blocking on the other hand is not illegal yet such as putting up buildings made of material that block out cell phone signals, that is, blocking the signals from ever getting to the cell phone. Blocking is legal because it is just shielding and does not interfere with any users external to the shielded areas. The concept of operation of the suggested system in this paper is that it will be operated in nonpublic areas that are private properties under the complete control of the keepers of the areas. The system design proposed in this work guarantees minimal or no spillage of RF power outside of the desired CA to avoid blocking any MSs that are outside the CA and in this respect can be viewed as one way of achieving passive blocking. The proposed system can also be operated in a connected mode, where the details provided (IMEI/IMSI) by an MS through a vBTS can be directed to a central server that is accessible by the MNO. All the decisions to control/release an MS are done by the MNO. The MNOs, through business agreements with the keepers of a CA, can allow/disallow certain services based on a particular attribute to provide or not to provide certain services (voice or data, e.g.) to selected MSs according to the whitelist, time of day, or location [8].

The remainder of this paper is organized as follows. In Section 2, the proposed system architecture and associated design are presented. In Section 3, the system prototype and performance evaluation are presented. In Section 4, simulation results are shown, while in Section 5 we draw our conclusions.

\section{Proposed System Architecture}

We propose a modification to the system that was presented in [8] by replacing the directional inner antenna with a set of leaky feeder antennas (i.e., radiating antennas) that are interconnected using a DAS connected to the inner vBTS [11]. The outer vBTS and the associated directional antenna that were described as part of the method of [8] are no longer necessary following the proposed modification.

In the system design, we used a GSM BTS transceiver vBTS from Lyrtech Inc. [18], which is a development system that can be used to develop small-scale, short-range, GSM BTS type of application for homes and small offices. However, any picocell BTS type can be used in the proposed system.

A set of leaky feeder antennas interconnected via a DAS is placed inside the CA to monitor and report on MSs that enter and/or leave the CA, as shown in Figure 1, where the vBTS unit is represented by the vBTS/vBSC (virtual Base Station Controller) entity. The vBTS is set up to clone the GSM (Global System for Mobile Communications) BTS within the MNO and uses controlled RF power. The set of leaky feeder 


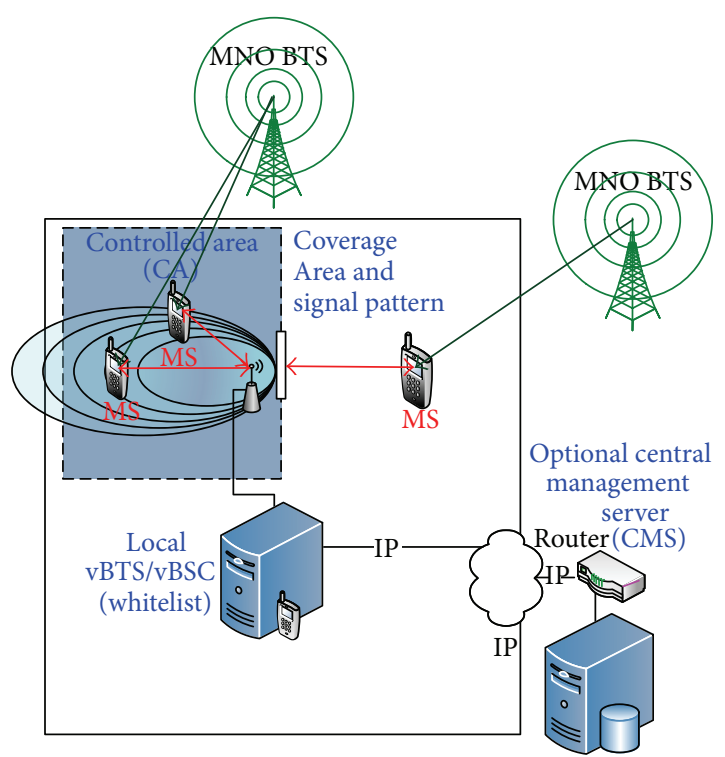

Figure 1: Architectural features of the system.

antennas are placed in the CA such that they can detect the identity of an MS when it enters the CA. If an MS is detected by the DAS via a leaky feeder, it is considered to be within the CA. For an MS to be controlled, it must be within the CA and must respond to paging signals from the vBTS via the DAS antenna. If a controlled MS is no longer detected by the DAS/leaky feeder combination, it is released (uncontrolled) from the system. This occurs naturally as the MS moves back under the influence of the MNO BTS signal when it moves away from the CA. The vBTS is hosted in the CA and is connected to the DAS using RF cables [11].

The placement of leaky feeder antennas in the CA is done in a way to achieve the best distribution of the vBTS signal using a set of multiple radiating cables to achieve the best coverage of the CA, while at the same time minimizing unwanted coverage outside the CA.

Within the overall system configuration, a CA is defined by a single local vBTS/vBSC group and the associated DAS rather than the two vBTSs/vBSCs and their associated directional antennas, as in the system proposed in [8].

A CA may optionally be connected to a Central Management Server (CMS) through a TCP/IP (Transmission Control Protocol/Internet Protocol) connection. The CMS would then be able to connect to all CAs that are deployed in a number of different locations. The CMS would receive all MS data collected from each CA for which a whitelist is defined. In the CMS, the MS data received from a CA, which are the IMEI and IMSI numbers, can then be checked against the whitelist for the specific CA to determine whether or not to control the MS.

In the system described in this paper, the CMS centralization was not implemented. The whitelist therefore was created and managed autonomously by the business logic software implemented at each CA.

To force an MS that enters the CA to connect to the vBTS installed in the CA, a "clone" signal (which is identical to the signal transmitted by the MNO BTS) that is stronger than the signal to which the MS was originally connected is transmitted by the DAS. This will attract the MS to connect to the vBTS that broadcasts the stronger signal, which in turn will force the MS to transmit its IMEI and IMSI information to identify itself and camp on the local vBTS. The vBTS will then be able to collect the required MS data and pass it through the BSC to the optional CMS server for verification and any further decision-making.

Control of the MS services in the CA is achieved by keeping the MS connected to the local vBTS at the CA and not providing any of the MNO services if the MS is not on the whitelist of allowed MSs. However, if the MS is on the whitelist, the local vBTS at the CA then releases the MS by denying its location update request during idle mode reselection. In this way, the MS reconnects to the MNO BTS and continues to function as it would normally [11].

In contrast to our previous work in [8], the business logic software simply checks the whitelist and attempts to match the IMEI/IMSI combination that identifies the user. Unlike the previous system [8], the system proposed in this paper does not rely on the detection steps of the control signals transmitted from MS. In other words, it does not require each MS to be attracted by an "outer" vBTS followed by an "inner" vBTS. This means that the system is easier to implement and is less dependent on management of the 3GPP (3rd Generation Partnership Project) protocols that control idle mode reselection. The system takes the actions that are described in Table 1.

It should be noted that in [8] if an MS goes through the two directional antennas at the gateway to the CA in an OFF state and is subsequently turned $\mathrm{ON}$ inside the CA, that MS will not be controlled even if it was not included in the whitelist since the system would not have considered it to be inside the CA. In contrast to that, in this work, if the MS has entered the controlled area by any entrance in an OFF state and was subsequently turned $\mathrm{ON}$ inside the CA, then it will still be managed when the leaky feeder and the signal strength are optimally configured.

We define the concept of a Gray Area (GA) as the area outside the CA where unwanted signal power spillage from the vBTS is high enough to cause, or indeed maintain, undesirable control over MSs that are located outside the CA as shown in Figure 2. One of the main advantages of the use of leaky feeders in this work, when compared with the use of directional antennas as per [8], is that they allow better and more precise management of this GA. In our evaluation of the proposed system, mitigation of undesired effects on MSs in the GA is one of the performance evaluation metrics that we use to measure the success of our design.

It should be noted that the GA also existed in our previous design which was presented in [8], but it was less predictable in that case because of the use of directional far field antennas reflections and fading. For this reason, the signals from the "inner" and "outer" antennas could result in incorrect MS classification (i.e., a mobile phone located outside the desired CA is undesirably controlled).

In contrast, leaky feeders can be deliberately designed to ensure that any signals that spill outside the CA are very 
TABLE 1: Available actions that can be taken based on current state and CMS rules.

\begin{tabular}{lcccc}
\hline Case & Current state & First rule & \multicolumn{2}{c}{ Second rule } \\
MS in whitelist & Action \\
& & Detected by vBTS & No & Control \\
2 & Uncontrolled & Yes & Yes control & Control (maintain) \\
3 & Uncontrolled & Yes & No & No control (release) \\
4 & Controlled & Yes & Yes & Yes
\end{tabular}

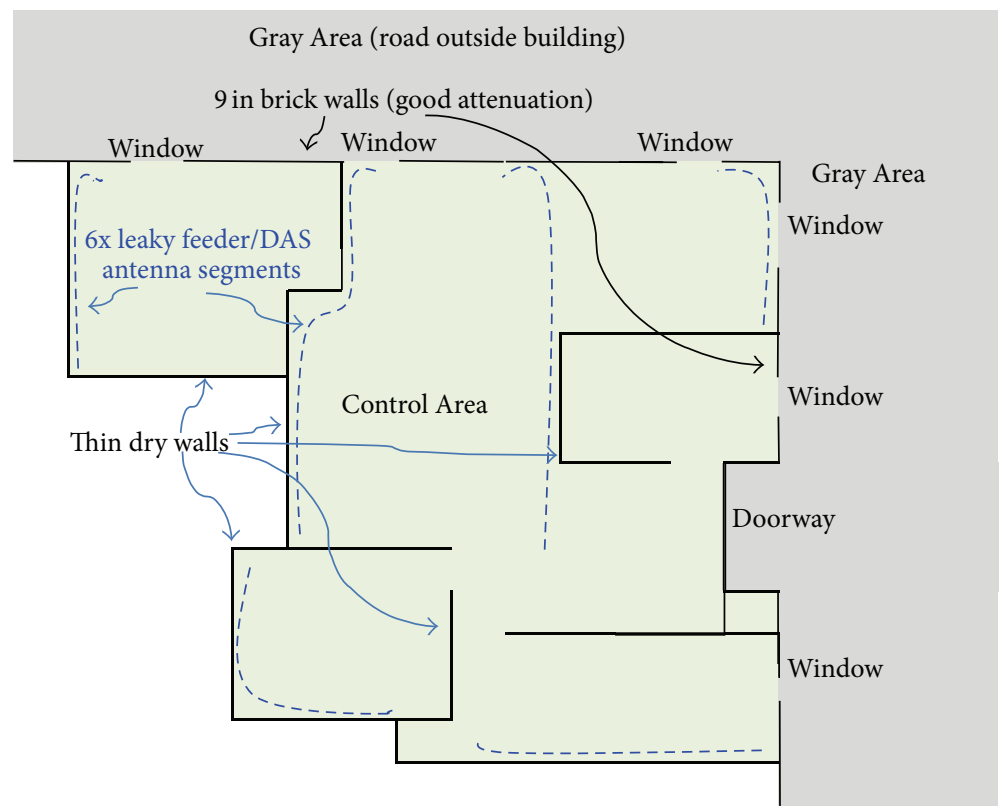

Figure 2: The CA floor plan and the leaky feeder antenna.

weak and are less likely to attract any MSs outside the CA to connect to the vBTS. Thus, undesired control of external MSs is reduced while full control is obtained over MSs in the CA.

\section{System Prototype and Performance Evaluation}

In order to deploy the proposed system in an area, a radio survey must be conducted within the CA to measure the $\mathrm{RF}$ signal levels and other parameters that are required to setup the system. In particular, these parameters will be used to configure the vBTS to mimic the operation of an MNO BTS. Each MNO BTS broadcasts using one or more fixed Absolute Radio-Frequency Channel Numbers (ARFCNs). The following Broadcast Control Channel (BCCH) parameters are monitored for analysis and for selection of a cell to be cloned: the $\mathrm{BCCH}$ Allocation (BA) list, the Location Area Code (LAC), the Cell Reselection Offset (CRO) value, the Cell Reselect Hysteresis (CRH) value, the Calculated Cell (C2) reselection value, the Mobile Network Code (MNC), Mobile Country Code (MCC), the Network Color Code (NCC), the Cell ID (CID), and the Base Station Identity Code (BSIC). These values are received in System Information (SI) messages that are broadcast periodically on the $\mathrm{BCCH}$ by the various BTSs. The MS is only allowed to attach to a BTS that meets specific conditions, which are described in detail in [8].

The parameters and information collected must then be analyzed to determine the appropriate clone values that must be used to configure the vBTS. This process is usually conducted when a new CA is commissioned and can be repeated periodically thereafter when significant changes are observed in the surrounding RF environment.

To set the boundaries to attract/release an MS to/from the system (thereby defining the vBTS coverage limits), the $\mathrm{C} 2$ with the $\mathrm{CRO}$ and $\mathrm{CRH}$ values can be configured in the vBTS as shown in Figure 3. To attract an MS to the vBTS in the system, we must configure the $\mathrm{C} 2$ value of the vBTS to be higher (e.g., by $12 \mathrm{~dB}$ ) than the sum of the $\mathrm{C} 2$ and $\mathrm{CRH}$ values of the serving MNO BTS. For the MS to be released from the system or to exit from the CA, it must switch from the vBTS to an MNO BTS that has a higher $\mathrm{C} 2$ value than the sum of the $\mathrm{C} 2$ and $\mathrm{CRH}$ values of the vBTS. This process occurs naturally by idle mode reselection when the MS leaves the CA.

The CA that was used in the measurement had the following specifications: an office space, approximately $13 \mathrm{~m}$ wide by $14 \mathrm{~m}$ long, which also had some internal dry walls, as shown in Figure 2. The CA is externally bounded by $0.225 \mathrm{~m}$ thick brick walls on the top and right sides, where external 


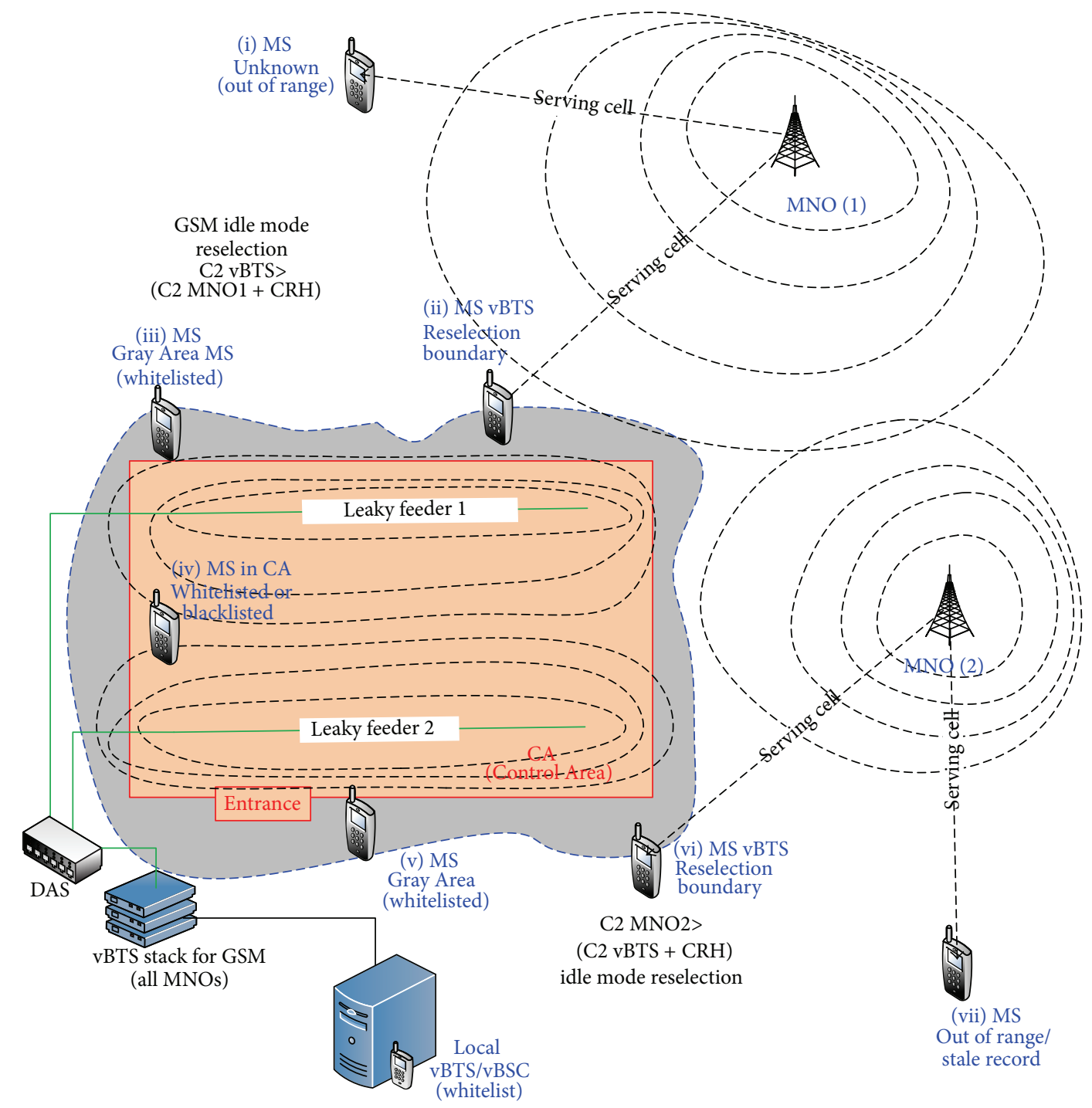

FIgURE 3: Effects of C2 and CRH values on attracting/releasing an MS to/from the system using leaky feeders.

access to the GA was also available for measurements. The left and bottom walls were of the dry wall type and were not effective for attenuation of RF signals, so the measurements were focused on the sides with brick walls. The bounding of a CA with brick walls is considered beneficial for both attenuation of MNO BTS signals inside the CA and also attenuation of vBTS signals outside the CA, which helps to minimize the GA.

The CA floor plan is shown in Figure 4 and is divided into $1 \mathrm{~m}$ square blocks. It was not possible to survey every block as a number of these blocks were occupied by furniture or shelves and not accessible for our measurements. Thus, seven representative grid blocks composed of 12 blocks each were surveyed (e.g., from 1_1 up to 1_12 and ranging from 7_1 up to 7_12). Similarly, in the GA, some representative rows or columns of $1 \mathrm{~m}$ blocks were marked out for survey (e.g., from $8 \_1$ up to $8 \_12$ and ranging from $12 \_1$ up to $12 \_12$ ). The Nemo MS used for the test was placed on a wooden shelf approximately $1.2 \mathrm{~m}$ above the floor to create consistent test conditions. Careful selections of each vBTS transmitter (Tx) power setting and the $\mathrm{CRO}$ value are made inside the $\mathrm{CA}$, so that the vBTS $\mathrm{C} 2$ value is approximately $12 \mathrm{~dB}$ higher in the CA than that of the MNO.

In that configuration, the leaky feeders are mounted approximately $10-15 \mathrm{~cm}$ away from the wall at a height of $2 \mathrm{~m}$. Note that the top and right-hand side walls are $0.225 \mathrm{~m}$ thick brick walls with a few windows but the left-hand and bottom walls are of dry wall construction. The GA considered in the survey is at the top and at the right outside the brick walls, which provide good RF attenuation.

Nemo Handy test MS [19] was used to survey each block in the CA and GA described in Figure 4 to measure the C2 difference values. It was used instead of power meters because it provides the net result of attraction probability for an MS based on the actual C2 average measurement and resulting calculation done by a typical GSM-type phone for idle mode reselection. Therefore, whether there is downlink fading/interference and so forth from MNO or leaky feeder 


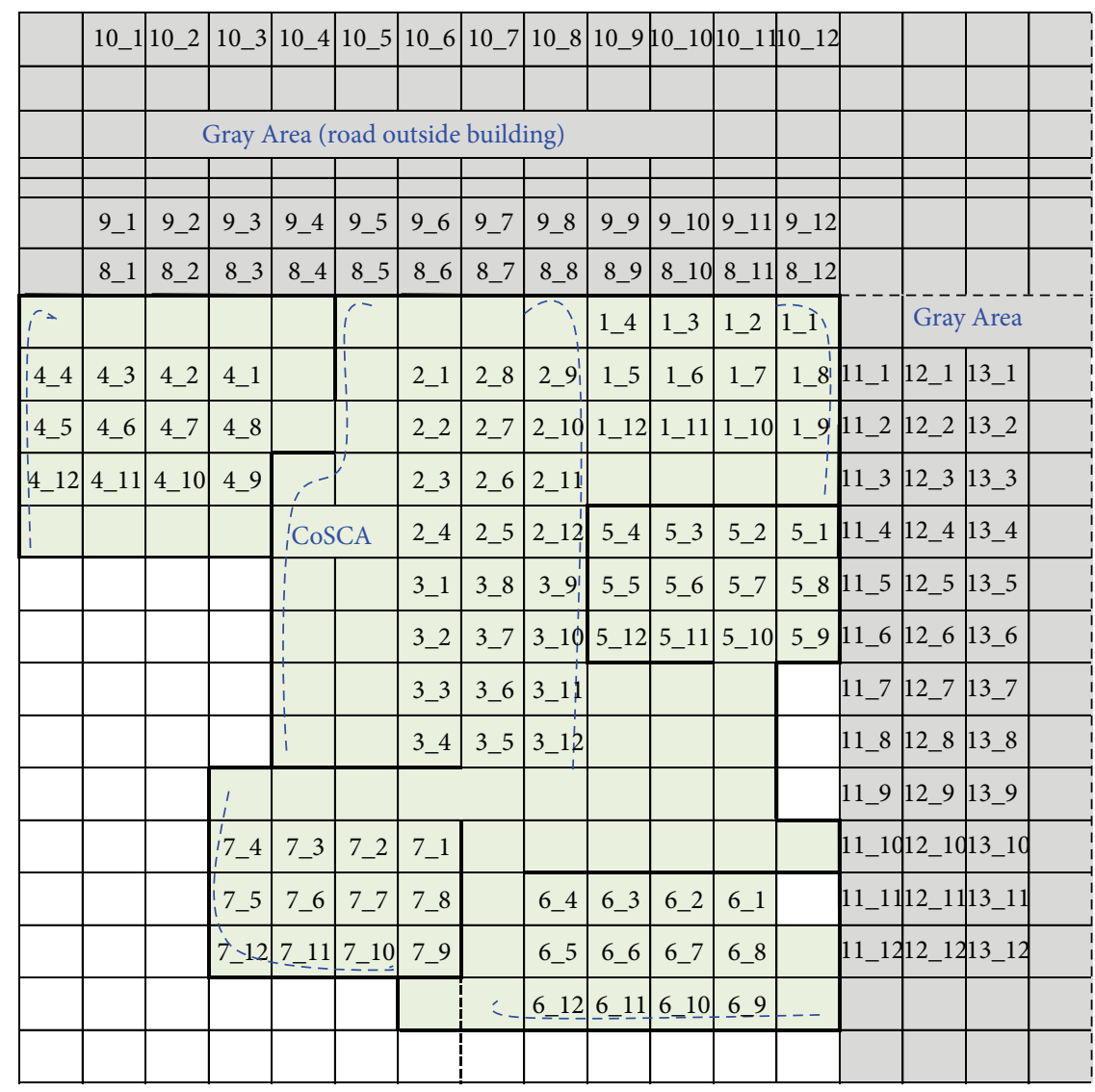

FIgURE 4: The CA and GA survey blocks.

in the final analysis, it is the MS that makes the decision by comparison between the averaged measured/calculated C2 of the GSM vBTS and the C2 of the relevant MNO BTS on which the MS is attached. When the C2 difference value is positive it should attract, when it is negative it should not attract (ignoring CRH hysteresis which can be used to affect this).

The $\mathrm{C} 2$ difference values and the physical mapping for the CA shown in Figure 5 indicate the results of this measurement. A high positive C2 (red color) value indicates stronger attraction to the vBTS than a low positive value. Normally, a negative $\mathrm{C} 2$ difference value (blue color) would result in MNO BTS selection by the MS rather than vBTS selection.

In the measurements, the $\mathrm{C} 2$ difference value between the serving cell and the first neighbor cell is shown (measured in $\mathrm{dB}$ ); that is, if the vBTS is not a serving cell or a neighbor cell, we would therefore neglect it and consequently will not have a $\mathrm{C} 2$ value for it that can be used to calculate the $\mathrm{C} 2$ difference between the BTS and the vBTS in that location. In the analysis, the $\mathrm{C} 2$ value of the MNO BTS is consistently subtracted from the vBTS C2 value. This simplification does not materially affect the results. It should be noted then that the value "none" shown in the GA in Figure 5 is most likely to be an "MS is not controlled" position because the vBTS was not ranked first or second in the neighbor list (i.e., received power from the vBTS in that location is very weak).
This is a fairly typical case, showing good CA coverage with significantly lower influence in the GA. For example, the C2 difference values shown in Figure 5, Block 4 (11.0, 15.1, 11.7, $3.0,6.6,11.6,14.8,14.6$, and $7.3 \mathrm{~dB}$ ), indicate likely attraction by the vBTS (desirable for non-whitelist MS control) and the $\mathrm{C} 2$ difference values $(-3.1$ and $-5.7 \mathrm{~dB})$ indicate likely attraction by the MNO BTS (which is not wanted). These local differences may be attributed to RF fading and so forth, but the adverse effects can be reduced in the real world by adding some CRH (i.e., hysteresis) to the vBTS settings (probably around $6 \mathrm{~dB}$ ).

In the GA, the opposite situation is desirable, which means that negative $\mathrm{C} 2$ difference values are required to prevent MSs from being attracted to the vBTS. Thus, as shown in Figure 5, Blocks 8 and 9, only the positive $(4.4,1.2$, and $3.6 \mathrm{~dB}$ ) values indicate possible attraction to the vBTS (which is an unintended consequence), while all other values show probable attraction to the MNO (required for other MSs control to ensure that such MSs remain attached to the MNO).

In the measurements above, in 67 out of the total 84 (79.8\%) $1 \mathrm{~m}$ square blocks in the CA, the C2 difference values are positive and therefore successfully attracted by the vBTS, while the remaining 17 (20.2\%) were undesirably attracted by the MNO BTS. On the other hand, in 40 out of the total $48(83.3 \%) 1 \mathrm{~m}$ square blocks in the GA, the C2 difference 


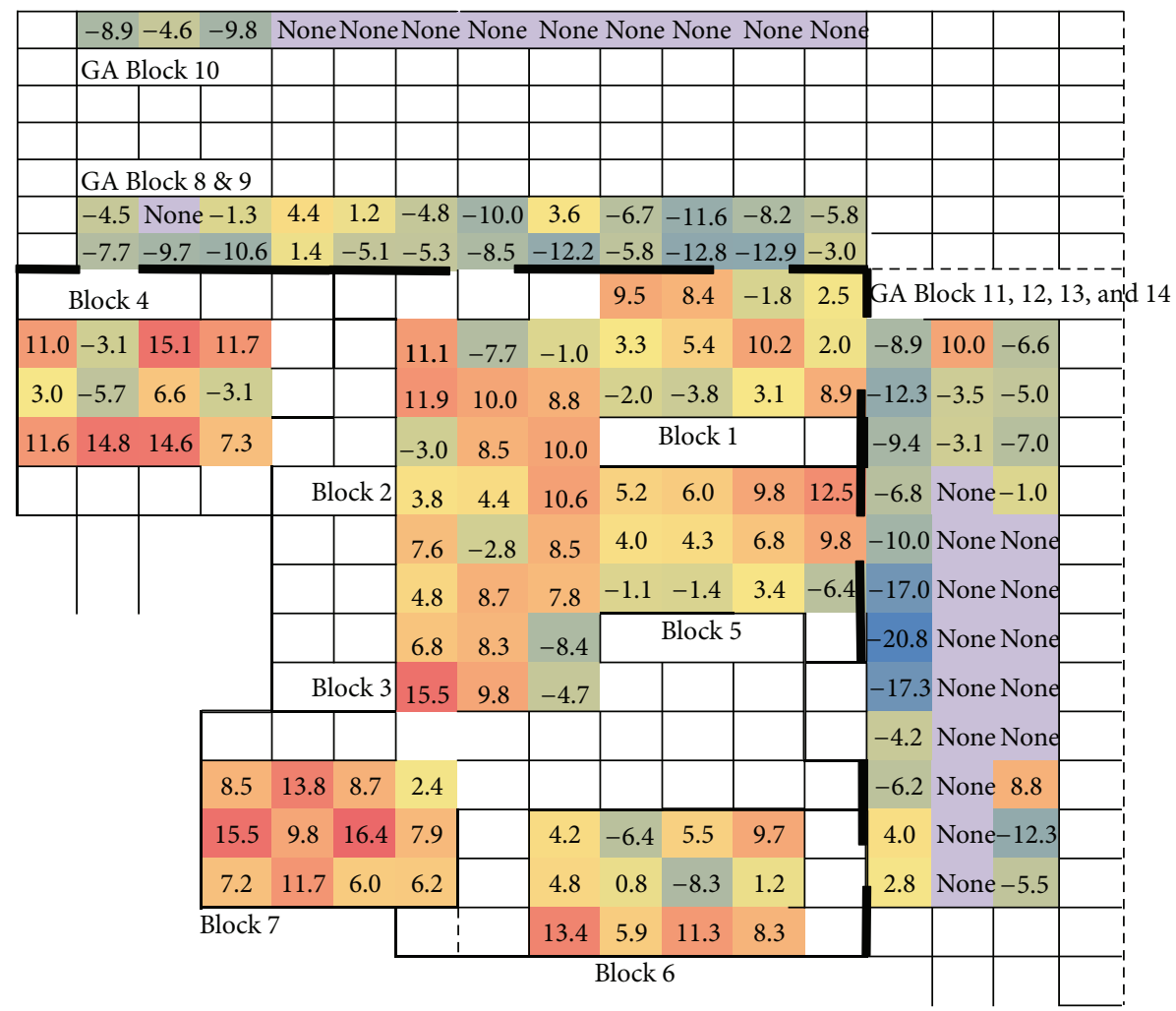

FIGURE 5: The (serving-1st neighbor) C2 difference results obtained for a single MNO in the DCS1800 band.

values are negative and therefore successfully not attracted by the vBTS, while the remaining $8(16.7 \%)$ were undesirably attracted by the vBTS.

Note that variation by as much as $15 \mathrm{~dB}$ in the RSSI signal levels is sometimes seen between adjacent test positions, even when they are only $1 \mathrm{~m}$ apart, which is probably caused by fading. Importantly, this behavior was observed even if the vBTS and the leaky feeders were switched off and only the MNO signals were monitored. It is shown that good coverage and control were achieved using the leaky feeder system despite this variation, but there is always a trade-off between control in the CA and reduced spillage into the GA. The GA shown is thus managed quite effectively using the leaky feeder array under the prescribed conditions.

\section{Simulation Results}

To compare the effectiveness of the DAS leaky feeder configuration versus the directional antenna configuration as described in [8] and to study different DAS leaky feeder placements we simulated the antenna radiation patterns using CST antenna design tool.

Simulations of antenna radiation patterns for both patch directional and leaky feeder antennas are illustrated in Figure 6. The radiation patterns from the simulations are used to simulate the received vBTS power in each $1 \mathrm{~m}$ square block.

These antennas were placed in the CA in different placements as shown in Figure 7. In Figures 7(a)-7(c), three different CA floor plan placement configurations for DAS
TABLE 2: Results of simulation in the CA and GA.

\begin{tabular}{lcccc}
\hline \multirow{2}{*}{ Parameter } & \multicolumn{3}{c}{ Leaky feeder } & Directional antenna \\
& $A$ & $B$ & $C$ & 79 \\
\hline MSs held in CA & 83 & 81 & 81 & 5 \\
MSs not held in CA & 1 & 3 & 3 & 16 \\
MSs held in GA & 2 & 6 & 8 & 32 \\
MSs not held in GA & 46 & 42 & 40 &
\end{tabular}

leaky feeder (placements $A, B$, and $C$ ) are shown, while Figure 7(d) shows the patch directional antenna placement in the CA floor plan. Based on these simulations, we were able to compute the $\mathrm{C} 2$ difference values for all DAS leaky feeder placements and also for the directional antenna case.

We simulated a scenario where we have 84 MSs in the CA and 48 MSs in the GA such that we have 1 MS in every $1 \mathrm{~m}$ square block as shown in Figure 4. We then computed the number of MSs in the CA that were successfully attracted by the vBTS. We also computed the number of MSs in the GA that were undesirably attracted by the vBTS.

The aforementioned simulation scenario was repeated for the directional antenna configuration as well as for the three different leaky feeder placements shown in Figure 7. The results of the simulations are shown in tabulated form in Table 2.

When comparing the 3 different leaky feeder placements $A, B$, and $C$, we can see that placement $A$ yields the best attraction in the CA while maintaining the lowest attraction 


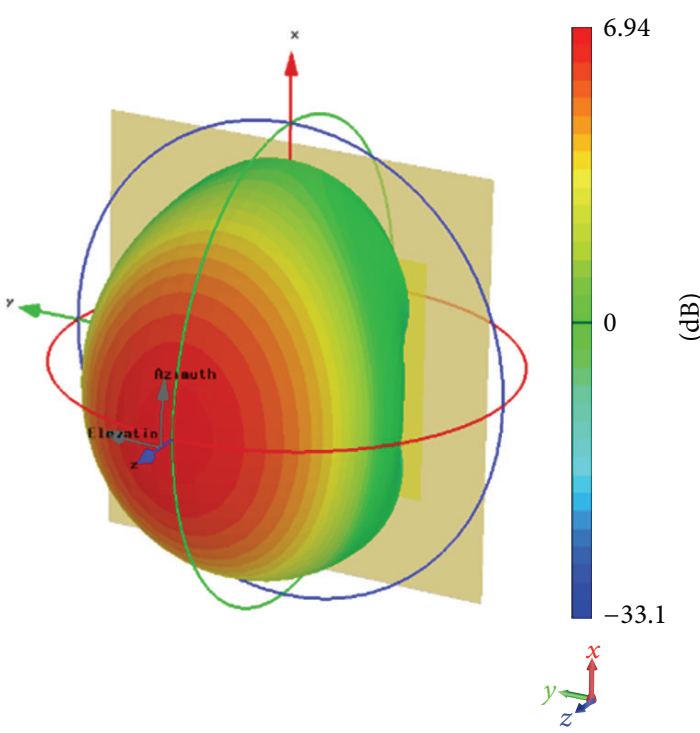

(a)

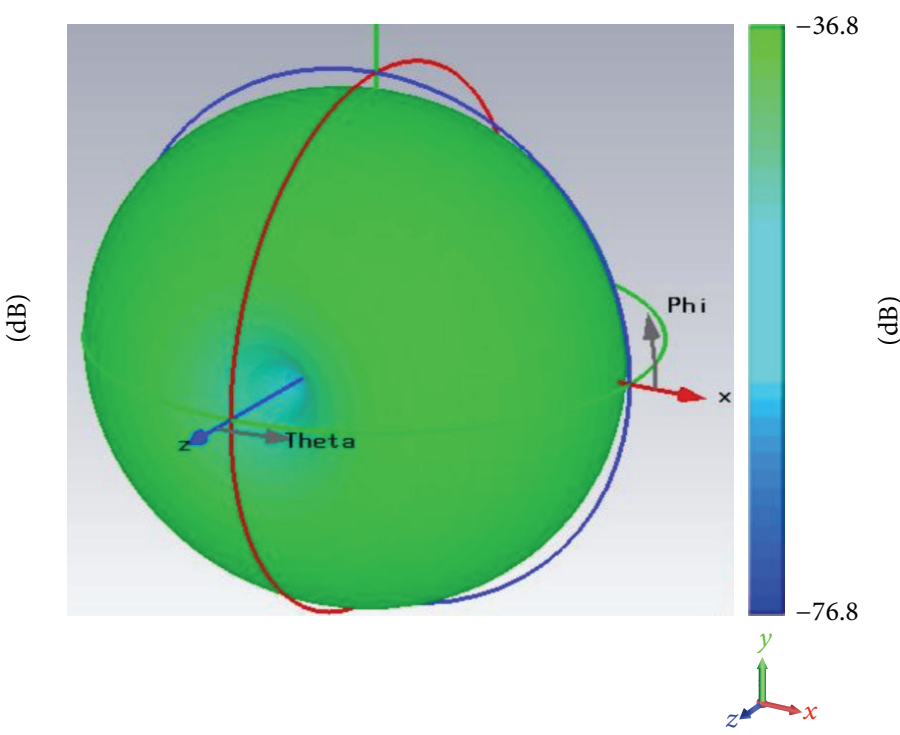

(b)

FIGURE 6: (a) Directional antenna radiation pattern. (b) Radiation pattern of one leaky feeder antenna.

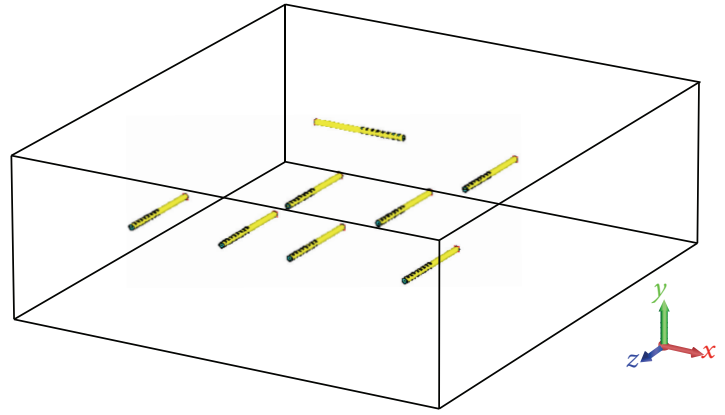

(a)

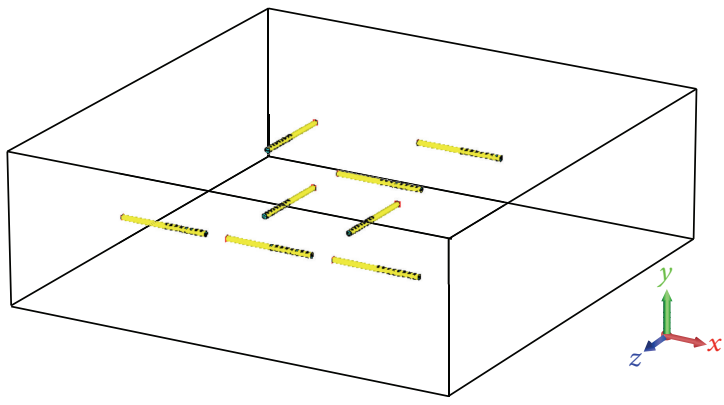

(c)

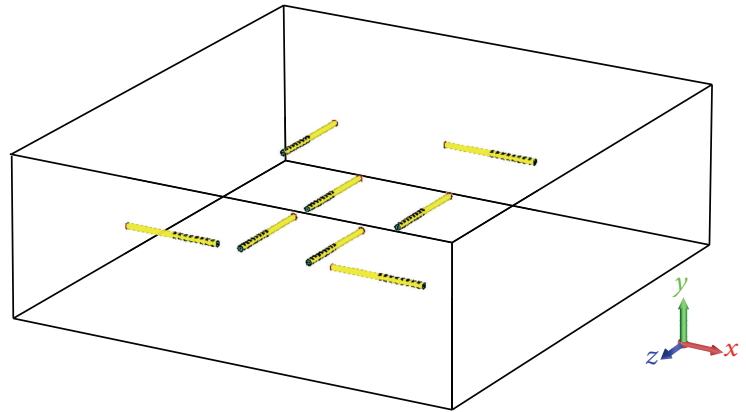

(b)

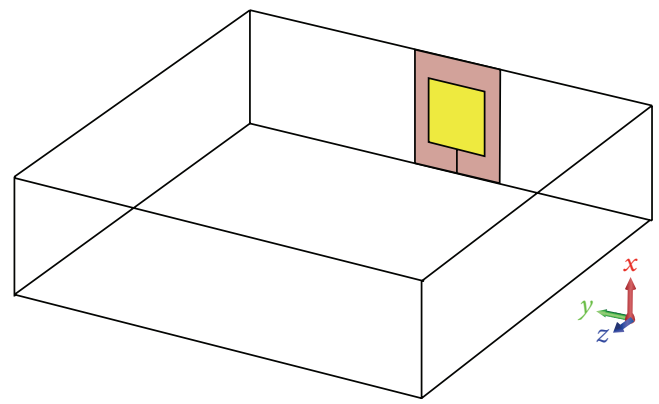

(d)

Figure 7: (a), (b), (c) Leaky feeder antenna placements $A, B$, and C. (d) Directional patch antenna.

in the GA. Placements $B$ and $C$ show the same performance in the CA but placement $B$ is slightly better in the GA as shown in Table 2.

In Table 2, we also show attraction results based on simulations using directional antennas as described in [8]. When comparing these results to any of the leaky feeder placements, all different placements of DAS leaky feeder considered in the simulation outperform the directional antenna in terms of number of MSs held by the vBTS in the CA. Although the directional antenna can provide a full coverage of the CA if its transmit power is increased, the number of MSs held by the vBTS in the GA can be very high. On the other hand, the placement of the leaky feeder can be designed to provide excellent coverage in the CA, while minimizing the undesired coverage in the GA, which yields a much better performance. 


\section{Conclusions}

In this paper, we proposed a system for controlling mobile communications services in areas where complete silence is either expected or mandatory, including schools, universities, places of worship, hospitals, and prisons. We proposed the use of leaky feeder antennas in the controlled area and show the superior performance of this system in controlling mobile services provided to MSs located within the CA, while mitigating unwanted RF signal power spillage outside the CA. This consequently prevents undesired control of mobile services that are provided to MSs outside the CA and minimizes the size of the GA.

In our future work, we shall find ways to ensure that newly released MSs can still be attracted by the vBTS and controlled by our system. It is important to extend the system to support UMTS/LTE. 3G/4G MSs can be captured on UMTS/LTE and then moved to GSM by the vBTS system using an inter-RAT (inter-radio access technology) handover mechanism for subsequent management in GSM, as described in this paper. Because of the additional security measures in UMTS/LTE systems, it is not possible to manage 3G MSs entirely in the UMTS/LTE domains.

\section{Conflict of Interests}

The authors declare that there is no conflict of interests regarding the publication of this paper.

\section{Acknowledgment}

This work was supported by King Abdulaziz City for Science and Technology under Project Design and Development of Mobile Station Class of Service System, part II, National Science, Technology and Innovation Plan (NSTIP) Grant no. 32-691.

\section{References}

[1] S. Pinyapong, H. Shoji, and T. Kato, "Mobile information service adapted to social, temporal and dynamic situational requirements of individuals," in Proceedings of the 2nd IEEE Asia-Pacific Services Computing Conference (APSCC '07), pp. 517-520, Tsukuba, Japan, December 2007.

[2] A. S. Acampora and M. Naghshineh, "Control and qualityof-service provisioning in high-speed microcellular networks," IEEE Personal Communications, vol. 1, no. 2, pp. 36-43, 1994.

[3] E. Efstathiou, The Mobile-Phone Silencers Controversy, Athens University of Economics and Business, Department of Computer Science, Mobile Multimedia Library, 2002, http://mm .aueb.gr/technicalreports/2002-MMLAB-TR-01.pdf.

[4] G. Antonini, A. Orlandi, and S. D'Elia, "Shielding effects of reinforced concrete structures to electromagnetic fields due to GSM and UMTS systems," IEEE Transactions on Magnetics, vol. 39, no. 3, pp. 1582-1585, 2003.

[5] M. Abu-Amara and M. Mahmoud, "Method of non-intrusive control of mobile device," USPTO Pub. No. US 2008/0113657 A1, 2008.

[6] J. Hong and A. Bilstad, "Methods for ring tone suppression informing and for informing a cellular telephone with configuration data," USPTO 7668557, 2010.
[7] J. Hsu and R. D. Regan, "Controlling device functions of a mobile terminal in a restricted area," USPTO US20130225086 A1, 2013.

[8] H. M. Behairy, W. S. Alrobian, A. A. Alghammas, and B. R. Suter, "Selective mobile communication in socially sensitive environments," Wireless Personal Communications, vol. 77, no. 4, pp. 3143-3157, 2014.

[9] H. Behairy, S. Alhumaidi, W. Alrobian et al., "Controlled mobile communication in a socially sensitive environment," USPTO 8346241, 2013.

[10] H. Behairy, S. Alhumaidi, W. Alrobian et al., "Controlled mobile communication as a service," USPTO 8428574, 2013.

[11] H. Behairy, W. Alrobian, A. Alghammas, Y. Alsuwayyeh, and F. Alqunaieer, "Controlled mobile communication as a service in a coverage area bounded by radiating cables," USPTO 8700026 , 2014.

[12] V. P. A. Santos, F. J. B. da Fonseca, L. J. de Matos, W. D. T. Meza, G. L. Siqueira, and L. A. R. Ramirez, "Indoor signal coverage of a leaky feeder cable," in Proceedings of the 15th SBMO/IEEE MTT-S International Microwave and Optoelectronics Conference (IMOC '13), pp. 1-5, Rio de Janeiro, Brazil, August 2013.

[13] https://apps.fcc.gov/edocs_public/attachmatch/DA-11-250A1 .pdf.

[14] http://ec.europa.eu/growth/sectors/electrical-engineering/rttedirective/.

[15] http://laws-lois.justice.gc.ca/eng/acts/R-2/FullText.html.

[16] https://www.comlaw.gov.au/Series/F2005B00449.

[17] http://www.citc.gov.sa/English/Pages/default.aspx.

[18] http://www.nutaq.com/.

[19] Nemo Handy - the world's most widely used handheld drive test tool, http://www.anite.com/businesses/network-testing/ products/. 

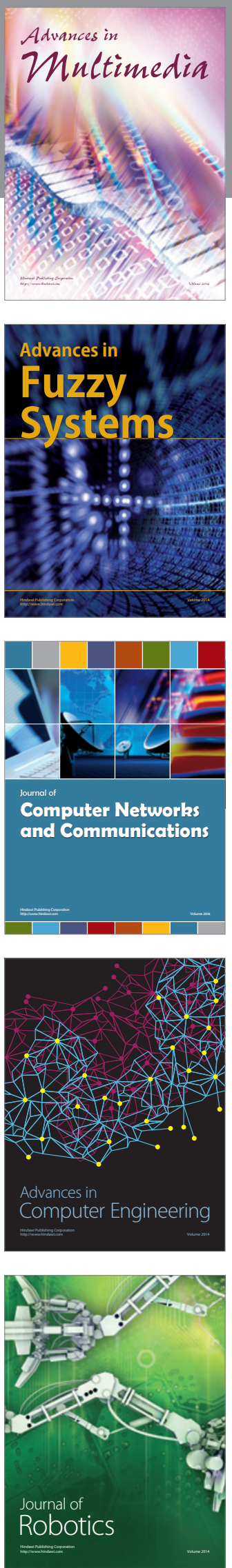

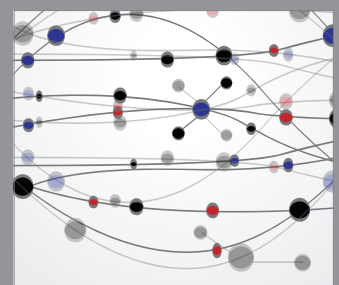

The Scientific World Journal
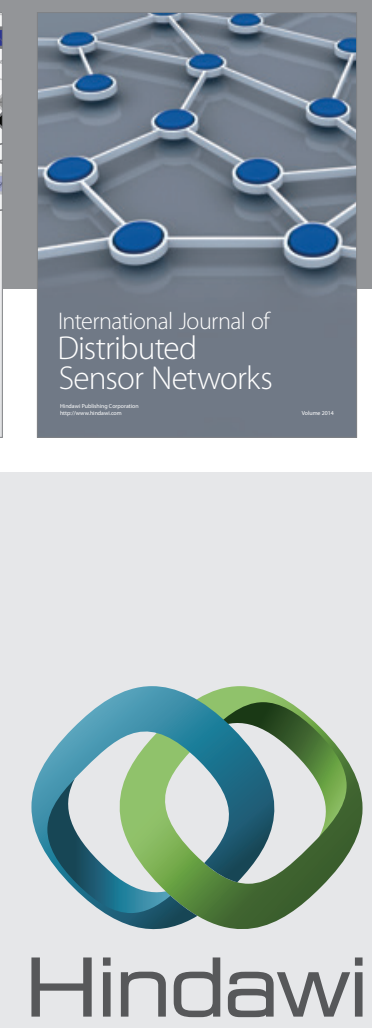

Submit your manuscripts at

http://www.hindawi.com
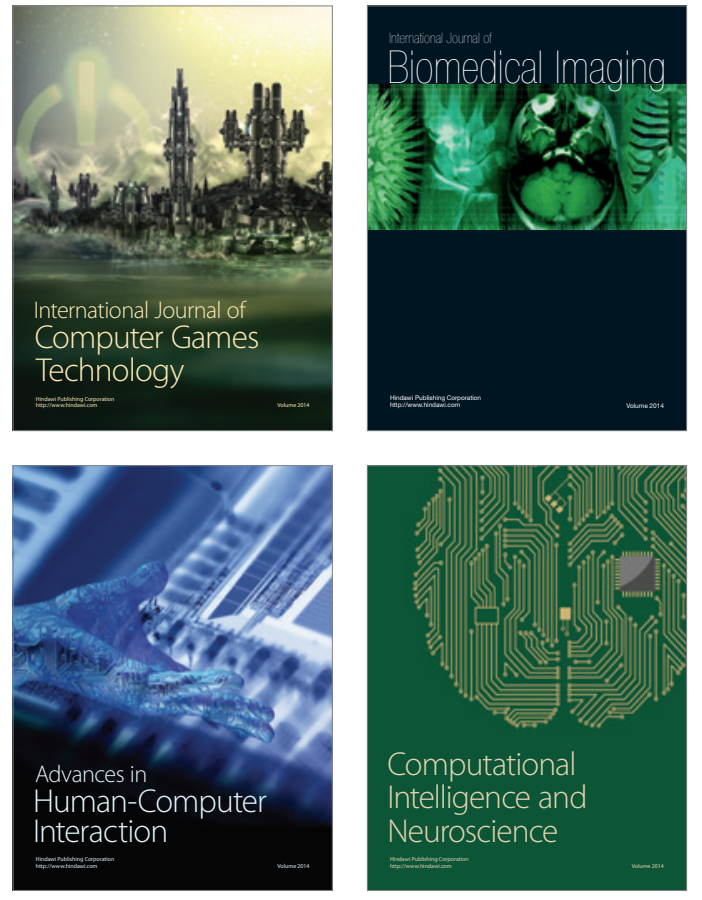
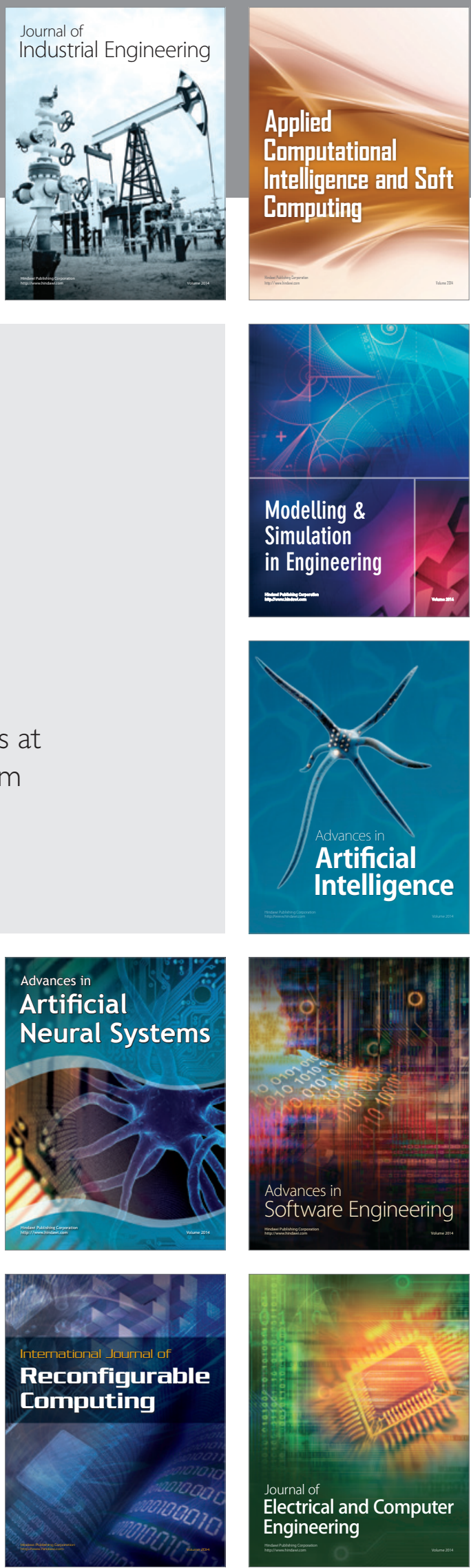